PAPER • OPEN ACCESS

\section{Resonant enhancement of Raman scattering by surface phonon polaritons in GaAs nanowires}

To cite this article: Sergey I Rybchenko et al 2021 J. Phys. D: Appl. Phys. 54475111

View the article online for updates and enhancements.
You may also like

Operando Raman Spectroscopy to
Understand the Reaction Mechanisms of
LiNi ${ }_{5.5} \mathrm{Mn}_{4} \mathrm{O}_{4}$ in Li-lon Batteries
Lucien Boulet-Roblin, Mouna Ben Yahia,
Daniel Streich et al.
- Determination of Phonon Deformation
Potentials in Carbon-doped Silicon
Kazutoshi Yoshioka, Ryo Yokogawa,
Tatsumi Murakami et al.
Determining Strain, Chemical
Composition, and Thermal Properties of
Si/SiGe Nanostructures Via Raman
Scattering Spectroscopy
Leonid Tsybeskov, Selina A. Mala, Xaolu
Wang et al.




\title{
Resonant enhancement of Raman scattering by surface phonon polaritons in GaAs nanowires
}

\author{
Sergey I Rybchenko ${ }^{1, *}$ (D), Sarfraz Ali $^{1}$, Yunyan Zhang ${ }^{2,3}$ and Huiyun Liu ${ }^{2}$ (D) \\ ${ }^{1}$ Department of Engineering, University of Hull, Hull HU6 7RX, United Kingdom \\ ${ }^{2}$ Department of Electronic and Electrical Engineering, University College London, London WC1E 7JE, \\ United Kingdom \\ ${ }^{3}$ Department of Physics, Universität Paderborn, Warburger Straße 100, 33098 Paderborn, Germany \\ E-mail: s.i.rybchenko@hull.ac.uk
}

Received 8 June 2021

Accepted for publication 3 August 2021

Published 16 September 2021

\begin{abstract}
Surface optical phonons are normally considered as subtle and poorly reproducible features in the Raman spectra of nanostructured semiconductors, from which little or no information about the sample can be extracted. The present study demonstrates the potential for changing this situation. For a common type of GaAs semiconductor nanowire (NW), we have shown that due to a combination of size-resonant light concentration, tapered shape and favourable scattering geometry, the surface phonon polariton (SPhP) Raman signal can be enhanced by orders of magnitude. The high signal gain enables routine detailed characterisation of the SPhP peak on an individual NW level, revealing its polarisation properties and spectral shift under variation of the dielectric environment. This detailed characterisation was conducted using very low excitation power density despite high absorption of the excitation light in the NW material. The findings provide an effective way to use SPhP Raman scattering in the characterisation of dielectric NWs and the prospect of developing novel surface sensors.
\end{abstract}

Supplementary material for this article is available online

Keywords: semiconductor nanowires, surface phonon polaritons, Raman scattering, dielectric photonic resonances, surface sensors

(Some figures may appear in colour only in the online journal)

\section{Introduction}

Surface phonon polaritons (SPhPs) are quasiparticle excitations existing on the surfaces and at the interfaces of noncentrosymmetric dielectric crystalline materials [1, 2]. They represent mixed optical-phonon-photon states, which are

* Author to whom any correspondence should be addressed.

Original content from this work may be used under the terms of the Creative Commons Attribution 4.0 licence. Any further distribution of this work must maintain attribution to the author(s) and the title of the work, journal citation and DOI. characterised by a direct optical response in the far infra-red (IR) spectral window, known as the reststrahlen band. The real part of the material dielectric permittivity is negative over the reststrahlen band, which leads to the near-surface localisation of the electric fields associated with SPhPs and their strong sensitivity to the dielectric properties of the surrounding environment [1]. This makes SPhPs attractive for crystal surface diagnostics and applications in surface sensors of local dielectric properties or as proximity detectors. When a crystal surface is in contact with (or in close proximity to) other materials or species characterised by atomic vibrations with energies appearing within the crystal's reststrahlen band, the interaction of $\mathrm{SPhPs}$ with these vibrations leads to strong enhancement of 
their optical response in IR and Raman spectroscopy [1, 3]. This creates another set of applications in the area of chemical sensing [4-7]. Recently, there has been a sharp increase of interest in SPhP or reststrahlen optics $[2,4,8,9]$ with the aim of developing low-loss SPhP photonic circuits. This greatly expands the range of possible applications for SPhPs and creates a demand for compatible far-IR optical components, such as sources, detectors and waveguides, especially in on-chip integratable form [10].

As a result, the role of experimental characterisation methods for SPhPs becomes even more important. Raman scattering and IR absorption-reflection spectroscopy have both been successfully used in the past, often in combination with specialised optical coupling methods based on prisms or gratings to study $\mathrm{SPhPs}$ on the surfaces and at the interfaces of bulk semiconductor and dielectric materials [1]. However, as far as nanostructures are concerned, traditional IR spectroscopy is severely limited. This is due to the low diffraction-limited resolution in the IR range, which makes visible-range-based Raman scattering in combination with confocal microscopy a more attractive option. Although new methods based on a combination of scanning near-field optical microscopy with nano-scatterers and waveguides have been demonstrated and actively used for the evaluation of the spatial properties of SPhPs [11], they lack the accuracy and simplicity of Raman scattering in characterising the energy spectrum and lifetime of SPhP excitations. Despite these advantages, examples of successful systematic observation of SPhPs in the Raman spectra of nanostructured materials are very rare. They include an earlier series on $\mathrm{GaP}$ nanoparticles [12] and reports on GaAs [13] and GaN thin films [14, 15]. More recently, a number of examples of experimental evidence for SPhPs were produced in the Raman spectra of semiconductor nanowires (NWs) [16, 17].

Semiconductor NWs are technologically mature 1D nanostructures that are commonly made of traditional ( $\mathrm{Si}, \mathrm{GaAs}$, $\mathrm{ZnS}$, etc) and also novel semiconductor/dielectric materials. They have novel optical and electronic properties that are not observed in other structures [18], and hence are being developed for a variety of applications in miniaturised photonics and electronics [19, 20], as well as in energy-harvesting devices [21]. Therefore, their photonic properties are being broadly studied.

Most often, weak but clearly distinguishable SPhP peak(s) have been reported in Raman spectra obtained from NW assemblies. The assemblies were either in the form of irregular arrays and 'haystacks' [22-24], scattered bundles [25] or regular vertically aligned arrays on a substrate $[26,27]$. In the case of well-characterised individual NWs, we are aware of only a few reports of well-resolved SPhP peaks in their Raman spectra (see [28-30]). The reason why the SPhP peak is shown in some reports but not routinely observed in other Raman studies of similar assemblies or individual NWs (see examples in [31] and [32]) remains unclear, precluding the development of any applications.

One possible reason for the elusive appearance of SPhP peaks in the Raman spectra of NWs is the apparent inefficient excitation of SPhPs in cylindrical geometry. From the polarisation point of view, an electric field polarised perpendicular to the NW surface is required for SPhP excitation [33, 34]. In practice, this means that the excitation light should be polarised perpendicular to the NW axis. However, this condition is known to lead to a severe attenuation of the excitation light intensity inside a thin NW, broadly cited as the 'antenna effect' [35]. However, in a more general picture of NW excitation, one should consider the specifics of light interaction with a cylindrical dielectric rod, when light can be admitted inside the cylinder in the form of so-called cylindrical waveguide modes with complicated polarisation patterns $[36,37]$. The electric field intensity in these cylindrical modes has a resonance character and can reach high values at specific NW diameters and excitation angles [38-40]. The most widely cited resonance condition, known as Mie resonance, has been broadly investigated analytically and via numerical simulations for typical semiconductors in both the cylindrical [41, 42] and spherical geometry [43]. However, the majority of these studies focus on light absorption efficiency, leaving the key question for Raman scattering, i.e. the matching of the polarisation and $k$ vector of the resonance modes to that of the phonons, largely unexplored. This is true even for the bulk phonon modes in the simplest spherical geometry [44]. To the best of our knowledge, theoretical studies predicting conditions for the amplification of the Raman signal from surface phonons at photonic (Mie and other) resonances are not available yet. Nevertheless, experimental evidence of Raman amplification on Mie resonances has already been demonstrated in InAs [45], silicon [46-48] and germanium NWs [49, 50], but only for the bulk phonon modes.

Here we demonstrate an amplification of the Raman signal of SPhPs via resonant light concentration in GaAs NWs, establishing the relevant experimental conditions and providing simulation-supported analysis.

\section{Methods}

\subsection{NW growth}

GaAs NWs were fabricated by solid-source molecular-beam epitaxy (MBE) with a solid Ga source and As4 cracker cell. B-doped silicon (111) substrates $380 \mu \mathrm{m}$ thick were used in the experiments. The NWs were grown with a Ga beam equivalent pressure, V/III flux ratio, substrate temperature, and growth duration of $1.12 \times 10^{-7}$ Torr, $44, \sim 630{ }^{\circ} \mathrm{C}$ and $1 \mathrm{~h}$, respectively.

\subsection{Sample processing}

In order to obtain NWs detached from the substrate (cut-off NWs), the as-grown sample was sonicated in ethanol using an ultrasonic bath $(60 \mathrm{~W})$ for $5 \mathrm{~min}$. The resulting dispersion of NWs in ethanol was subsequently applied in drops onto a $\mathrm{Si} / \mathrm{SO} x$ substrate to deposit the cut-off NWs. The sonicated substrates ('shaved' substrates) were removed from the ethanol and dried in a nitrogen gas flow. 
For the paraffin encapsulation, paraffin oil was applied as a drop on the surface of an as-grown sample. For silicone encapsulation, a drop of freshly prepared mixture of RTV630 (Techsil) resin and curing agent was placed on the as-grown sample and slowly squeezed into a $20-30 \mu \mathrm{m}$ thick film by a $200 \mu \mathrm{m}$ cover glass slide. The glass slide was removed after the RTV polymerisation was complete, leaving the RTV film on the sample surface.

\subsection{Raman measurements}

All Raman measurements were conducted at room temperature using a LabRAM (HORIBA) spectrometer equipped with a confocal microscope, Peltier-cooled charged-coupled device detector and HeNe laser $(\lambda=632.8 \mathrm{~nm})$. The microscope was equipped with a precise $X-Y$ scanning sample stage (minimum step of $0.2 \mu \mathrm{m})$, piezo focus lens positioner and $\times 100$ dry objective lens $(\mathrm{NA}=0.92)$. For mapping, the confocal diaphragm of the microscope was set at 1 Airy unit, resulting in optical resolution of the system of $0.27 \mu \mathrm{m}$ and $1 \mu \mathrm{m}$ for the in-plane $(X-Y)$ and vertical $(Z)$ directions, respectively. The excitation laser power was adjusted using a builtin set of reflective neutral density filters, and a power level of $12 \mu \mathrm{W}$ was selected to minimise the laser overheating (see supplementary figure S10, available online at stacks.iop.org/ JPD/54/475111/mmedia, and note S4 for details). Polarisation unanalysed Stokes-Raman spectra $\left(z\left(x^{\prime} x^{\prime}+x^{\prime} y^{\prime}\right) \bar{z}\right.$, or unanalysed spectra) were normally collected except where crossed $\left(z\left(x^{\prime} y^{\prime}\right) \bar{z}\right)$ or parallel $\left(z\left(x^{\prime} x^{\prime}\right) \bar{z}\right)$ polarisation is explicitly noted. For polarisation control, a half-wave plate and absorptive polariser were used. The results were corrected for the spectrometer instrumental polarisation function.

\subsection{Numerical simulation}

The numerical simulations were performed using the finite element method implemented in commercial COMSOL Multiphysics software. The results were calculated by solving Maxwell's equations in the frequency domain for a suspended NW and plane-wave excitation. The material optical parameters for GaAs have been taken from [51, 52]. NWs were simulated as either a $4 \mu \mathrm{m}$ or $2 \mu \mathrm{m}$ long cylinder or a truncated cone suspended in a large cavity bounded by absorbing Perfectly Matched Layers (COMSOL). When simulating the truncated cone shape, the top diameter is kept at half of the pedestal diameter.

\subsection{Analytical calculations}

Analytical calculations were performed to obtain the dispersion of electromagnetic modes in a dielectric infinite cylinder by solving equation (1) [53]:

$$
\begin{gathered}
{\left[\frac{1}{u} \frac{J_{n}^{\prime}(u)}{J_{n}(u)}-\frac{1}{v} \frac{H_{n}^{\prime}(v)}{H_{n}(v)}\right]\left[\frac{\varepsilon}{u} \frac{J_{n}^{\prime}(u)}{J_{n}(u)}-\frac{\varepsilon_{m}}{v} \frac{H_{n}^{\prime}(v)}{H_{n}(v)}\right]} \\
=n^{2} \frac{k_{z}^{2}}{(\omega / c)^{2}}\left(\frac{1}{v^{2}}-\frac{1}{u^{2}}\right)^{2}
\end{gathered}
$$

where $v=k_{\mathrm{m}} R, u=k R, n$ is an integer, and $J_{n}$ and $H_{n}$ are standard Bessel (first kind) and Hankel functions; $k_{z}$ denotes the mode complex wavevector along the cylinder axis at frequency $\omega . k$ and $k_{\mathrm{m}}$ are the transverse components of the mode wavevector inside and outside the cylinder, respectively:

$$
k^{2}=\varepsilon \frac{\omega^{2}}{c^{2}}-k_{z}^{2}, k_{\mathrm{m}}^{2}=\varepsilon_{\mathrm{m}} \frac{\omega^{2}}{c^{2}}-k_{z}^{2}
$$

The dielectric permittivities of the cylinder and embedding medium are $\varepsilon$ and $\varepsilon_{\mathrm{m}}$, respectively, with corresponding refractive indices $n=(\varepsilon)^{1 / 2}$ and $n_{\mathrm{m}}=\left(\varepsilon_{\mathrm{m}}\right)^{1 / 2}$. The cylinder material (GaAs) is assumed to be non-magnetic. Only guided modes (with $k_{\mathrm{z}}>\omega / c$ ) have been calculated. The calculations were done using commercial Matlab software.

\section{Results and discussion}

\subsection{Hot spots in Raman mapping}

GaAs NWs were fabricated by MBE with self-catalysed mode on silicon substrates. They have an average diameter of 50$70 \mathrm{~nm}$ and length of 4-6 $\mu \mathrm{m}$, with characteristics typical of the chosen growth method, which were described in detail in earlier publications $[54,55]$. At the substrate-NW interface (figure $\mathrm{S} 1$ ), there is a layer of aggregates with a thickness of 300-500 nm, known as 'parasitic clusters'. The clusters share the same crystallographic orientation as the NWs, but they have an irregular morphology.

Raman mapping of the as-grown GaAs NW samples revealed strong highly localised maxima (hot spots) that have an integral signal intensity exceeding that of the individual cutoff NWs (see section 2) by two orders of magnitude. A typical map of the integrated Raman signal taken with the objective focussed at $\sim 2 \mu \mathrm{m}$ above the substrate level (mid-height of NWs) is shown in figure 1(a) (see also figure S2(a)). The density of the hot spots varies across the sample, but it is always less than $10 \%$ of the average NW density. This Raman hotspot phenomenon is common for NW samples grown over the time span of a year.

A correlated study of the Raman maps and corresponding scanning electron microscope (SEM) images allows the origin of the hot spots to be established. Figures 1(b) and S2(b) show representative Raman maps overlaid on top-view SEM images of the same area of the samples. Zooming in on this composite map (figures 1(c)-(e), S3(a), (b) and S4(a), (b)) reveals that the Raman maxima are correlated with the distribution of thicker and tapered NWs. The geometrical parameters of these thicker NWs were obtained from their SEM images taken at an inclined view to the sample normal as shown in figures 1(f)(h) and S3(c)-(f). In total, 16 hot spots across two samples were characterised in this way and the mean half-height NW thickness at the hot spots was obtained as $110.7 \pm 21 \mathrm{~nm}$. This value is $1.5-2$ times larger than the diameter $(50-70 \mathrm{~nm})$ of the majority of NWs. The latter also do not show any prominent tapering. The length-averaged taper of hot-spot NWs is within the $0.3^{\circ}-0.7^{\circ}$ range. This taper corresponds to $\approx 2$ times diameter variation over the typical NW length of $3-5 \mu \mathrm{m}$. 

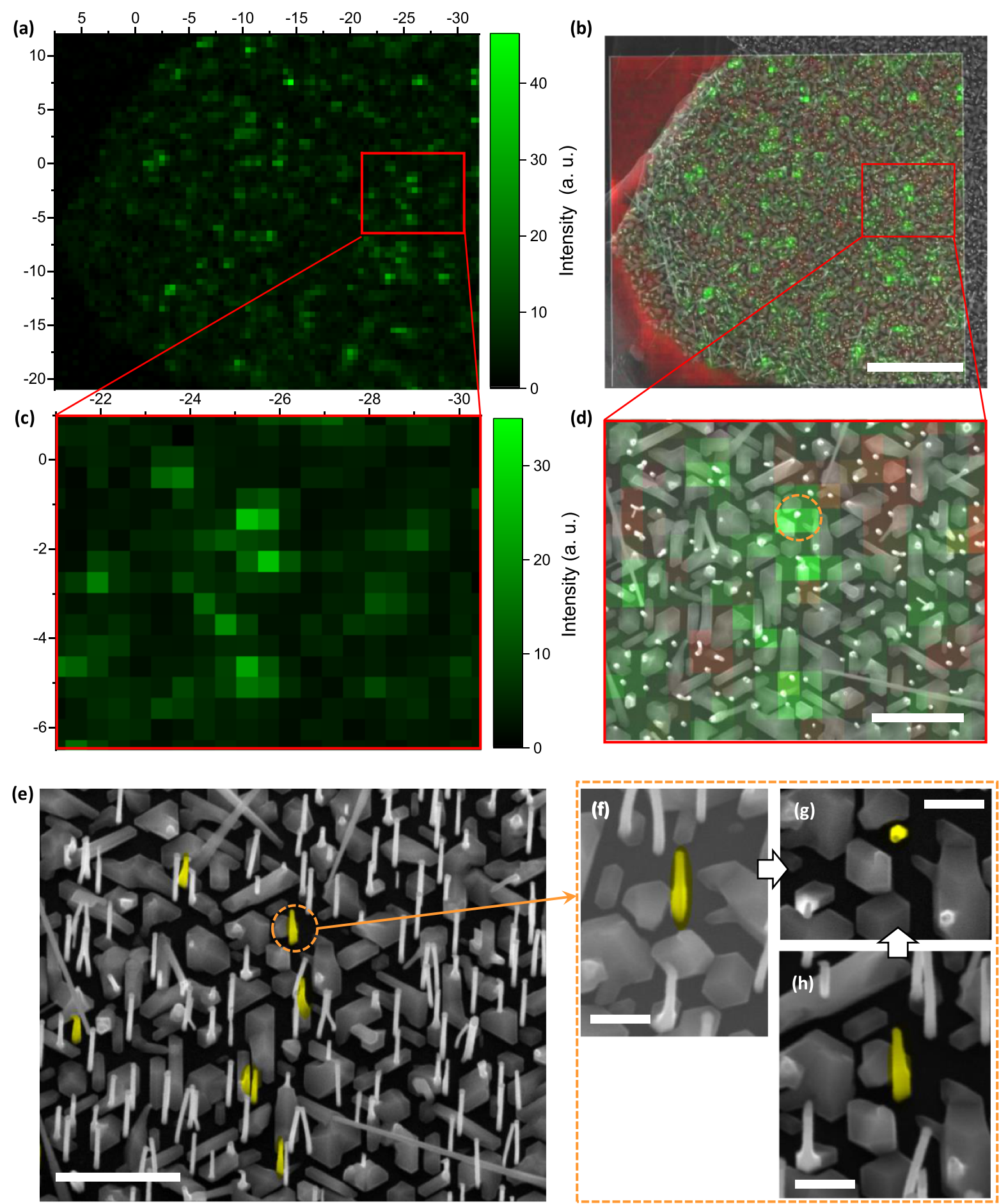

Figure 1. Raman map of as-grown GaAs NW sample. (a) Integral intensity map of GaAs phonon band. Scale is in $\mu$ m. (b) Same map as (a) (green colour) combined with mapping of Raman signal from the silicon substrate (red colour) and superimposed on SEM top-view image. Scale bar is $10 \mu \mathrm{m}$. (c), (d) Zoom into the Raman and composite maps shown in (a) and (b), respectively. Scale in (c) is in $\mu \mathrm{m}$ and scale bar in (d) and (e) is $2 \mu \mathrm{m}$. (e) SEM image of the same area as (d) taken at $10^{\circ}$ inclination. NWs highlighted in yellow are related to the brightest green spots in (c) and (d). (f), (g) SEM images of the selected hot-spot NW from (e) (encircled in orange) taken at different angles: (g) top-view; (f) view at $10^{\circ}$ inclination to the left side; (h) view at $10^{\circ}$ inclination to the down side. Same scale bar of $500 \mathrm{~nm}$ is applicable for (f)-(h). The selected NW is isolated from other NWs and characterized by slightly elongated cross-section, thickness $d \approx 107 \mathrm{~nm}$ at half-height, taper $\approx 0.8^{\circ}$, and height $\approx 3.1 \mu \mathrm{m}$.

The scattering geometry employed (figure 2(a)) allows for mapping along the NW height (length) on as-grown samples, adding a third dimension to the Raman map. From the 3D Raman mapping of the hot spots shown in figure 2(b) (also see figure S5), the maximum of the Raman signal is often located at the mid-height level of the NW, not at the thicker base as might be intuitively expected. This also implies that the Raman signal from the underlayer of thick clusters typically 

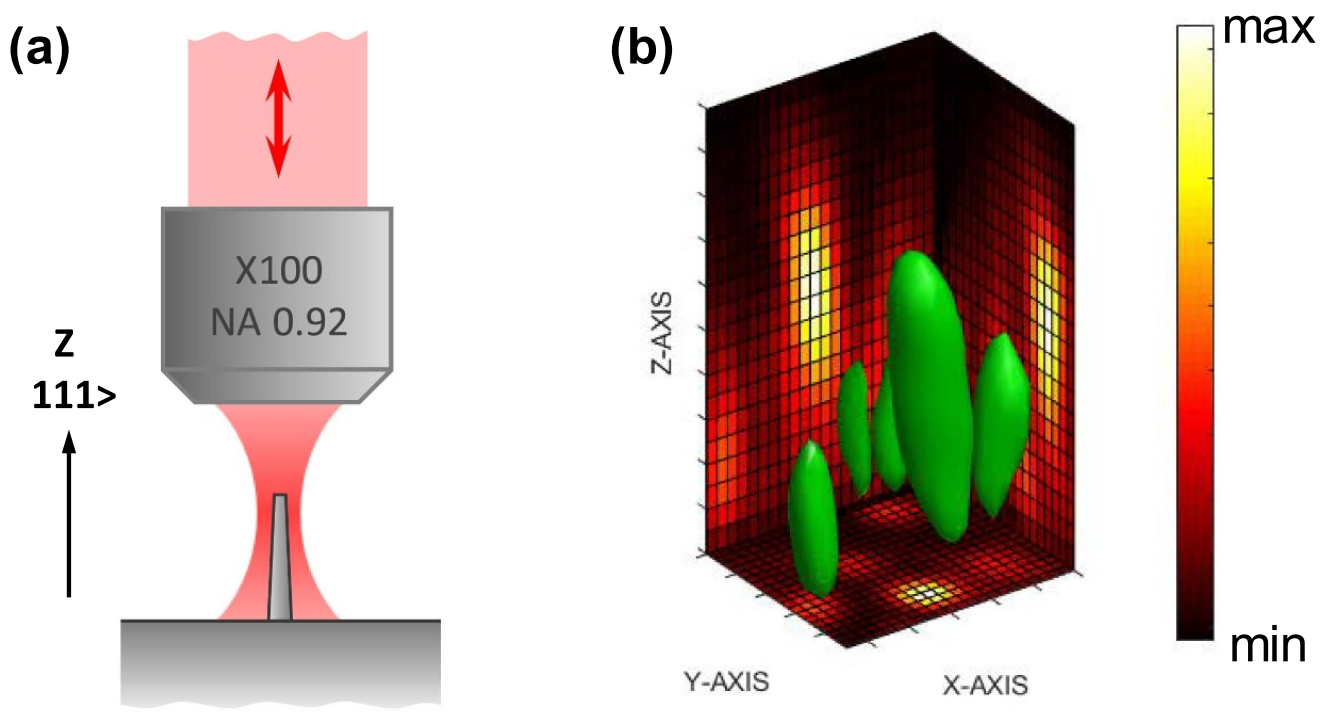

Figure 2. (a) Schematics of the scattering geometry used in experiment. (b) 3D map of integral Raman intensity of a group of hot spots. Green-colour isosurface (at $25 \%$ of max signal) is smoothed for clarity and shown together with maximum intensity projections in $x, y$ and $z$ directions. Scale division is $1 \mu \mathrm{m}$ for all axes. Bottom of $z$ scale is at $0.5 \mu \mathrm{m}$ below the substrate level.

present in our samples (figure S1) is much weaker than that of the hot spots. From the mapping of the 'shaved' samples (see section 2 and figure S1(b)), it was established that the Raman signal from the clusters is about an order of magnitude lower than that of the hot spots, despite the larger volume of material involved in the clusters. Moreover, the Raman signal magnitude from dozens of GaAs NWs glued into one standing up bundle (see figure S6(a)) remains about ten times lower than that of an individual hot spot. Thus, the magnitude of the hot-spot signal cannot be simply associated with the larger volume of the related NWs combined with the vertically standing orientation.

\subsection{Raman spectra of hot spots}

Hot spots are also characterised by an unusual Raman spectrum, different from that of the background (figures 3(a) and $\mathrm{S} 3(\mathrm{~g})$ ). Extra care has been taken to separate any overheating effects. For that, the spectra were recorded for an incident laser power range from 1 to $120 \mu \mathrm{W}$ and the heat-induced spectral line shift was used to select a suitable laser power (see figure S10 and note S4 for details). In general, the Raman spectra of the hot spots can be decomposed into three distinct peaks as shown in figure 3(b) (see also figures S3(h) and S7 and note S1). Two of them, identified as transverse (TO) and longitudinal (LO) optical phonon modes, have spectral positions at $269.2 \pm 0.5 \mathrm{~cm}^{-1}$ and $292.5 \pm 0.4 \mathrm{~cm}^{-1}$, respectively, which agrees well with bulk GaAs data $[56,57]$. According to the phonon theory of an ideal single-oscillator polar crystal, any modes in the frequency gap between bulk TO and LO modes are surface or interface related. Thus, the third dominant peak has been identified as a surface optical (SO) phonon mode, namely $\mathrm{SPhP}$ as confirmed below. Its spectral position varies within the $285-288 \mathrm{~cm}^{-1}$ range for different hot spots (figures 3(b) and S7). The full width at half maximum of the SO peak is $5.6 \pm 0.5 \mathrm{~cm}^{-1}$, which is significantly larger than the $\sim 3.5 \mathrm{~cm}^{-1}$ obtained for the TO and LO peaks (figure S7 and note S1). The SO peak is unique to the hot spots and is not observed in the spectra of clusters (figure 3(a)), NW bundles (figure S6(b)) or cut-off NWs (figure S8(b) and note S2).

The hot-spot Raman spectrum was investigated at different $z$ levels along the NW length (height) to probe the expected effect [34] of changing the NW thickness on the SO peak position. For that, two particularly long (tall) hot-spot NWs were selected and the spectra were collected at $2 \mu \mathrm{m}$ intervals, which is well above the $z$-axis resolution (see section 2) of our setup. As shown in figure 3(c) for one such hot spot, no prominent SO peak shift was detected in the spectra taken at different $z$ levels. A similar result was obtained for the second selected tall NW. At the same time, the SO intensity is sharply peaked in the middle part of the NW $(z=3-5 \mu \mathrm{m})$, similar to the integral signal distribution shown by the $3 \mathrm{D}$ map in figure 2(b). This suggests that both the overall Raman signal intensity and the SO peak position are defined by the relatively short 'bright' middle segment of the NW length. In the simulation section, we show that the appearance of the bright segment is related to the NW tapering.

The Raman spectrum of GaAs hot spots is often sensitive to the rotation of the laser polarisation in the substrate plane, and the hot-spot NWs displaying sensitivity to the polarisation rotation are characterised by an elongated cross-section (see figures 4(a), (b) and S4). The polarisation rotation strongly affects both the amplitude and position of the SO peak, while TO and LO peaks show moderate amplitude variations only. Specifically, a higher amplitude and spectral red-shift of the 

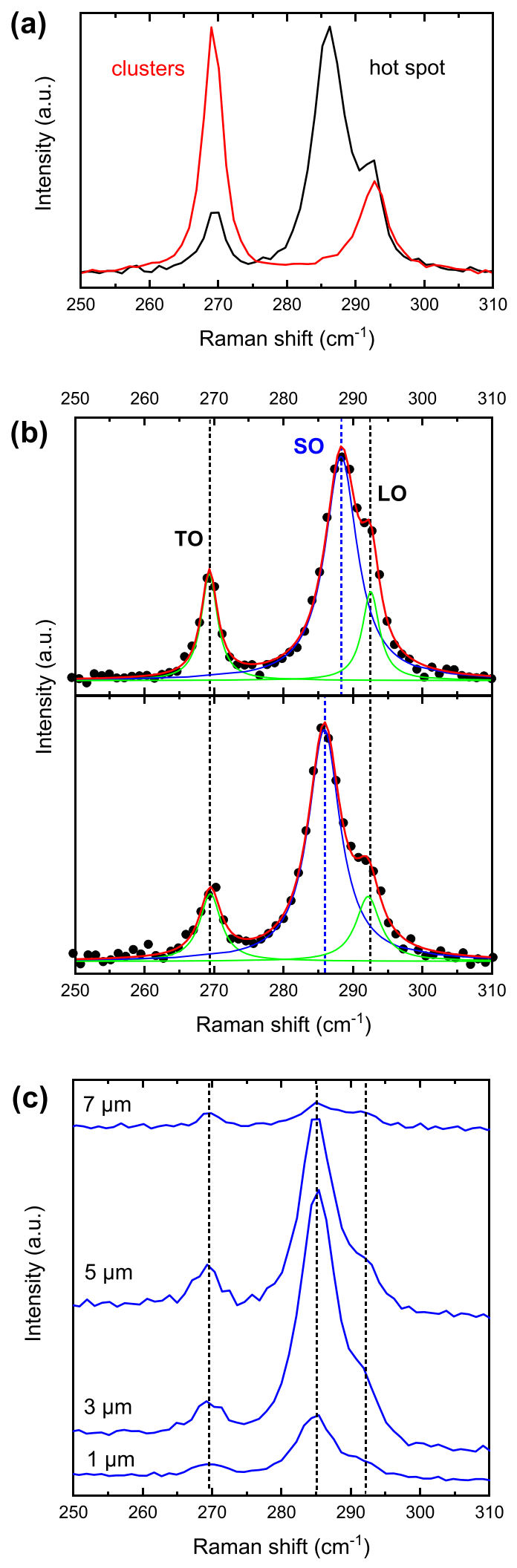

Figure 3. Raman spectra obtained on as-grown samples. (a) Normalised spectra of a hot spot and clusters. (b) and (c) Individual hot spots with blue-shifted (top panel) and red-shifted (bottom panel) SO peak position. Fitting by three Voigt peaks and peak assignment are shown as well. (c) Spectra taken on the same hot spot at different focal position $z(z=0$ at substrate). Spectra are offset for clarity.
SO peak is observed when the laser polarisation is aligned along the longer dimension of the NW cross-section (see figures 4(b), (c) and S4), which explains the variation of the SO spectral position between the hot spots (figure 3(b), figure S7). In hot-spot NWs with a round cross-section, the SO peak position was always found at $288 \pm 0.6 \mathrm{~cm}^{-1}$ (see figure 4(d) and (e), in air and figure S3). This behaviour of the SO peak in NWs with an elongated cross-section is different from the radius-dependent spectral shift observed in NWs with a round cross-section [25], and to the best of our knowledge it has not been discussed in the literature. It is analysed in detail in our follow-up paper [58], where it is explained by the shift and splitting of SO modes induced by the cross-section asymmetry.

A commonly accepted experimental test $[1,12,24]$ for the surface origin of the phonon peak in a Raman spectrum is its spectral shift under variation of the dielectric constant of the media surrounding the sample. Most often, paraffin oil (nujol) having an IR dielectric constant $\varepsilon=2.15$ has been used $[12,24]$. In the present study, the encapsulation (see section 2 for details) of NWs on as-grown samples within a thin layer of paraffin oil or polymerised silicone resin $(\varepsilon=2.0$, -2.5 [59]) has been employed. Figure 4(F) shows the Raman spectra averaged over several hot spots with presumed round NW cross-section (also see figure S9 and note S3 for details) taken before and after encapsulation. The comparison of these spectra shows a clear red-shift of $2-3 \mathrm{~cm}^{-1}$ of the SO peak (indicated by the blue arrow) under both paraffin and silicone encapsulations. Qualitatively, this result proves the surface origin of the assigned SO peak. The quantification of the encapsulation effect will be discussed later. Extra independent evidence for the surface origin of the SO peak has been obtained via the laser oxidation study (see figure S11 and note S4).

\subsection{Numerical simulations}

The experimental results indicate that the abnormal enhancement of the Raman scattering signal as well as the appearance of an SO (SPhP) peak is the result of the combination of the specific scattering geometry and morphological parameters of NWs. Excitation light couples with a NW in a highly inhomogeneous manner, which makes a standard Raman scattering picture based on plane wave interaction inapplicable. Instead, the approach based on the modal analysis of lightNW coupling has been employed. As a first approximation for high-aspect-ratio and lightly tapered NWs, the light propagation modes of an infinitely long non-absorbing dielectric cylinder have been considered, for which an analytical solution is available (see equation (1) in section 2). It is expected that some of these cylindrical modes could be excited by the laser beam in the excitation geometry employed (see figure 2(a)) in our experiments, leading to a high intensity of light field inside the NW and hence to strong enhancement of the Raman signal. For the excitation geometry with a laser beam directed parallel to the NW axis, the mode excitation efficiency cannot be predicted by analytical methods (in contrast to the case of Mie 
(a)

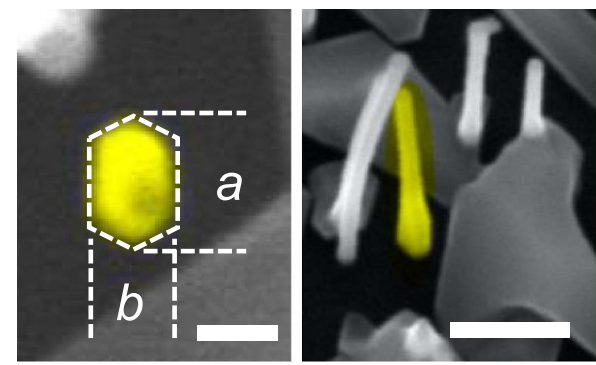

(b)

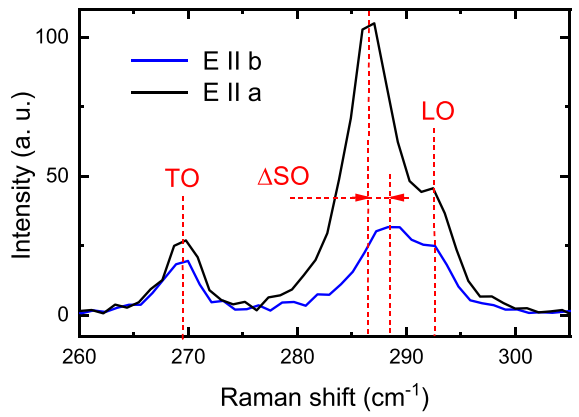

(d)
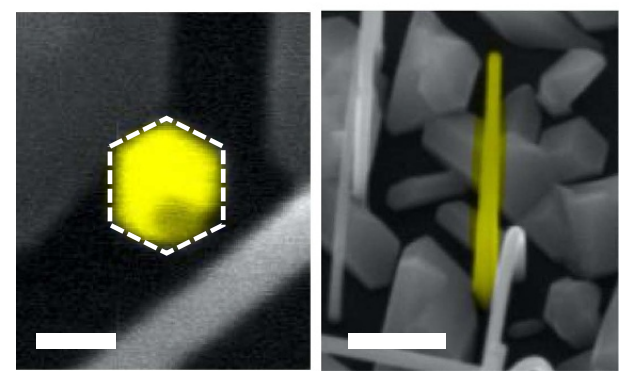

(e)

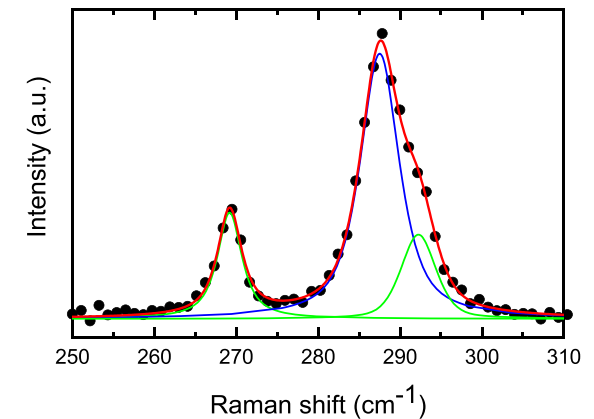

(c)

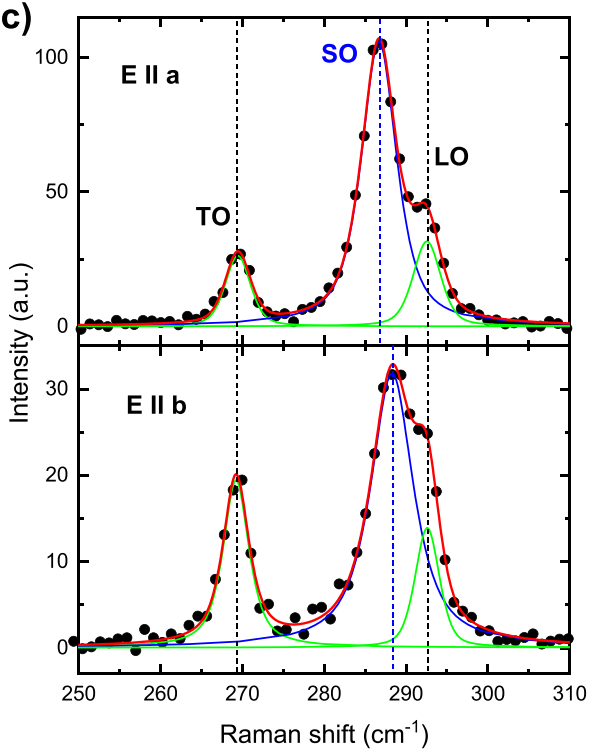

(f)

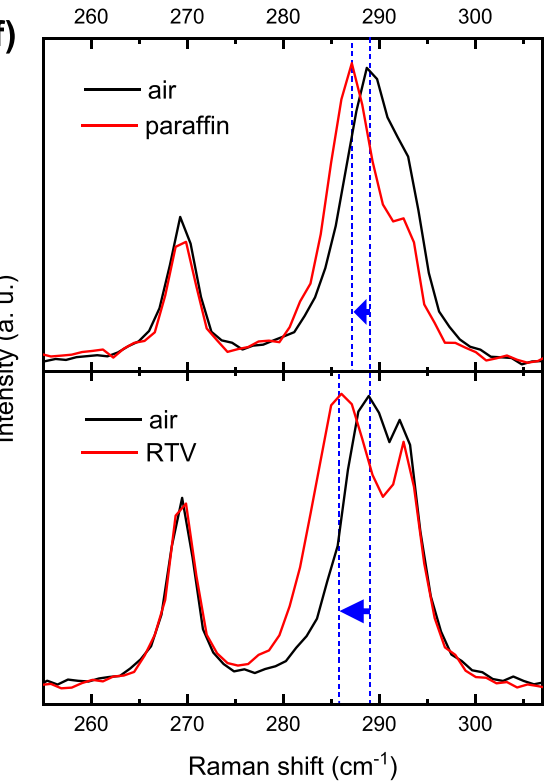

Figure 4. (a) SEM images of the hot-spot NW with elongated cross-section taken at normal (left) and inclined (right) viewing angle. Left image shows the cross-section parameters. Scale bar is $100 \mathrm{~nm}$ (left) and $500 \mathrm{~nm}$ (right). (b) Unanalysed Raman spectra of the hot spot from (a) at two perpendicular laser polarizations. The SO spectral shift between the spectra is indicated. (c) Fitting of spectra from (b) by Voigt peaks. (d) SEM image of the hot-spot NW of round cross-section taken at normal (left image, scale bar is $100 \mathrm{~nm}$ ) and inclined (right, scale bar is $500 \mathrm{~nm}$ ) viewing angle. (e) Unanalysed Raman spectrum of the hot spot from (d). (f) Normalised unanalysed Raman spectra illustrating the effect of paraffin (upper panel) and RTV (lower panel) encapsulation.

photonic resonances), hence a numerical simulation approach has been used.

\subsection{Photonic resonances}

From the simulation results, the volume-averaged excitation light intensity inside the GaAs cylinder was calculated as a function of the cylinder radius for a given laser wavelength of
$632.8 \mathrm{~nm}$ excitation and cylinder length of $2-6 \mu \mathrm{m}$. Figure 5(a) reveals a resonance-like behaviour with two peaks in the viable range of the NW radius, located at $52 \pm 1.5 \mathrm{~nm}$ and $130.5 \pm 1.5 \mathrm{~nm}$. The peak positions vary slightly with the NW length. More peaks appear if the radius is increased further (the next one is at around $215 \mathrm{~nm}$ and is not shown). Analysis of the electric field distribution (figure S12(a)) at the resonances revealed that peaks at $R_{\mathrm{C}} \approx 52$ and $\approx 130 \mathrm{~nm}$ are 
(a)

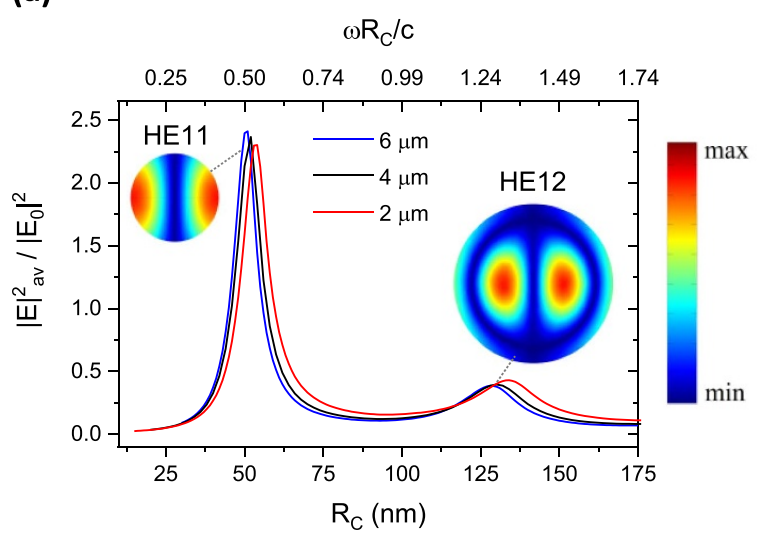

(b)

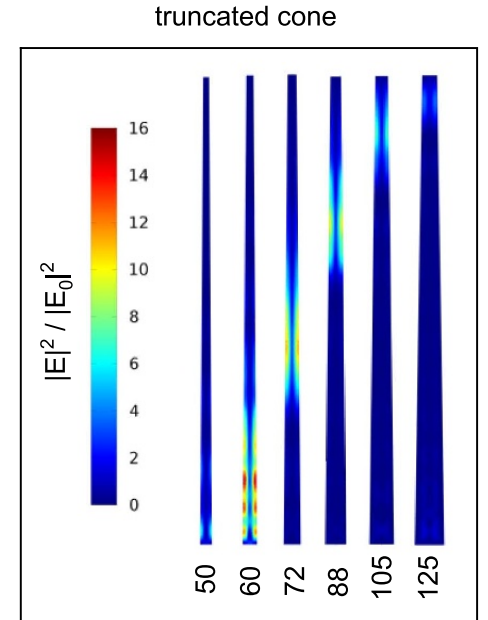

cylinder

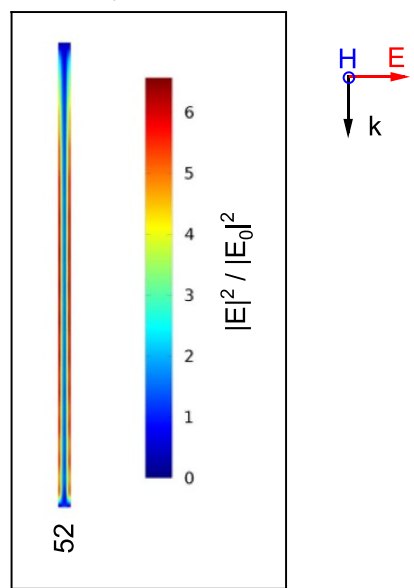

Figure 5. Simulated excitation field intensity. (a) Normalised average squared excitation electric field norm inside the GaAs and GaAsP cylinders of 2, 4 and $6 \mu \mathrm{m}$ length as a function of the cylinder radius. Inserts illustrate modal electric field distribution at the peaks. (b) Distribution of excitation field intensity along the axial cross-section of GaAs cylinder and cone at different pedestal radius (indicated at the bottom of each distribution). Insert illustrates excitation geometry.

associated with the excitation of HE11- and HE12-like cylindrical modes, respectively (resonances at higher radius values appear to be a continuation of the HE1X series). The peak in the $R_{\mathrm{C}}$ dependence of the average light intensity inside the NW can explain the selective enhancement of the Raman signal over a narrow part of the NW population. Indeed, the maximum average field for HE11 resonance in a GaAs cylinder is predicted to occur at a diameter $\left(2 R_{\mathrm{C}}\right)$ of $104 \pm 3 \mathrm{~nm}$, which is close to the $110.7 \mathrm{~nm}$ obtained for the mean midheight thickness of the hot-spot NWs. For quantification of the Raman signal enhancement, it is assumed that both the excitation and Raman Stokes components are described by the HE11 modes. The HE11 resonant peaks for the Stokes and laser wavelengths display strong overlap as shown in figure S12(b), which indicates that the related field distributions are very similar in both cases. Hence, ignoring the minor differences in the field distribution, the variation of the Raman signal can be approximated as a variation of the product of $I_{\text {laser }} \times I_{\text {Stokes }} \propto\left|E_{\text {laser }}\right|^{2} \times\left|E_{\text {Stokes }}\right|^{2} \approx\left|E_{\text {laser }}\right|^{4}$. In the $\left|E_{\text {laser }}\right|^{4}$ approximation, one immediately finds that the 20-30 times difference in the average field intensity predicted by figure 5(a) (see also figure S12(b)) between the resonant-diameter NW (hot spot) and the average-diameter NW $(50-60 \mathrm{~nm})$ translates into a 400-900 times difference in the Raman signal (per NW). This difference explains why the signal from the average-sized NWs on the Raman map of an as-grown sample is obscured against a background of much stronger signals from the hot spots and clusters.

The tapering adds a radiative character to the cylinder modes and hence facilitates the external light coupling to them [36]. To further investigate this effect in application to HE11-like resonance excitation, we have simulated the planewave excitation of the finite-length tapered GaAs cylinder. Specifically, a $4 \mu \mathrm{m}$ long truncated cone with the top radius two times smaller than the pedestal radius was chosen to mimic the experimental parameters of hot-spot NWs. The volume-averaged electric field intensity in this cone shows (figure S12(c)) peaked behaviour similar to that of the cylinder (figure 5(a)) but broadened due to the range of radiuses covered by each cone shape. The volume-averaged HE11 peak amplitude is similar in both cases, but the field distribution within the cone is much less homogeneous than in the case of the cylinder. Specifically, the light concentration tends to appear only over a part of the tapered length, where the local diameter is close to the resonant one (figure 5(b), left panel). This localised field intensity is significantly larger $(\approx 2$ times at the pedestal radius of $60 \mathrm{~nm}$ ) than the more homogeneous field in the case of the cylinder (figure 5(b), right panel). The extension of the light concentration zone depends on the extent of the taper (the stronger the taper, the shorter the bright zonenot shown). If the resonant diameter is not covered, the bright zone is not observed (see pedestal radius of $50 \mathrm{~nm}$ and $125 \mathrm{~nm}$ cases in figure 5(b), left panel). This means that the light concentration spot and hence the highest Raman signal can appear anywhere along the NW length (height), not necessarily at its thickest part. This explains the experimental observations of the maximum Raman intensity collected from the mid-height part of hot-spot NWs (see figures 2(b) and 3(c)).

\subsection{Wave vector of resonant mode}

The observation of efficient excitation of HE11-like modes for vertical illumination of cylindrical semiconductor NWs agrees with previous simulation studies, focussed on the light absorption efficiency [41, 42]. To aid the Raman scattering analysis, the excited modes should also be characterised by their wavevectors. By taking a Fourier transform (FT) of the simulated field distribution in the $z$ direction (along the NW axis), the modal $k_{z}$ vector values have been calculated from the FT frequency peak as illustrated in figure S13 (also see note S5). In figure 6(a), the $k_{z}$ values obtained for the 4 and $2 \mu \mathrm{m}$ long GaAs cylinders are plotted together with the guiding mode dispersion diagrams of an infinite non-absorbing GaAs cylinder as a function of the cylinder radius. The infinite 

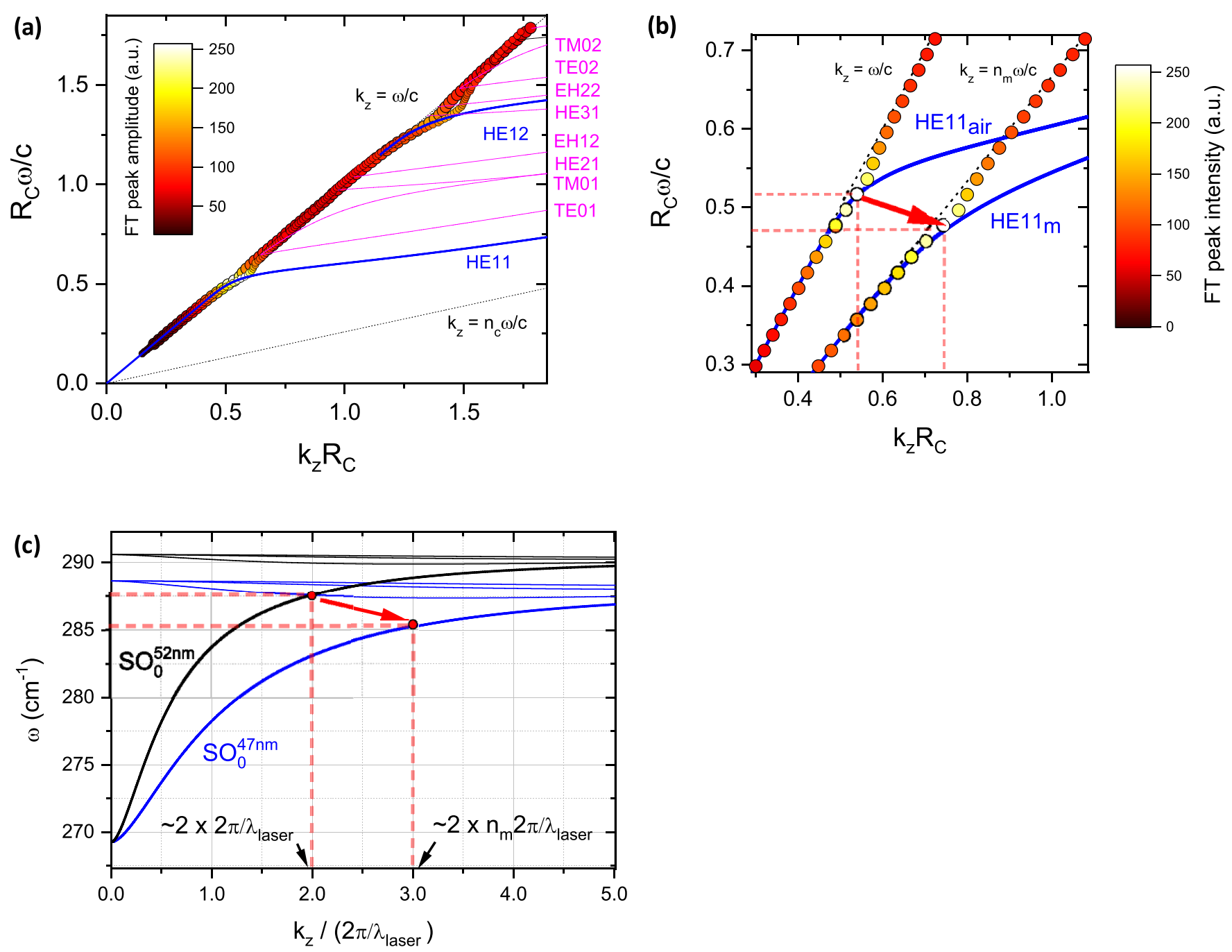

Figure 6. Simulated dispersion diagrams. (a) Solid lines: dispersion of waveguiding modes of infinite non-adsorbing GaAs cylinder at $633 \mathrm{~nm}$ excitation in air. Large (small) symbols: dispersion of the excitation electric field in $2 \mu \mathrm{m}(4 \mu \mathrm{m})$ long GaAs absorbing cylinder. Symbol colours indicate the FT peak intensity. Dashed lines indicate the light line in air and in GaAs. (b) Solid lines: dispersion of waveguiding modes of infinite non-adsorbing GaAs cylinder at $633 \mathrm{~nm}$ excitation in air and in paraffin/RTV media. Symbols: dispersion (FT) of the simulated excitation electric field in $4 \mu \mathrm{m}$ long GaAs absorbing cylinder placed in air and in paraffin/RTV media. Symbol colours indicate the FT peak intensity. (c) Dispersion diagram for the first four SPhPs of non-adsorbing infinite GaAs cylinder of $R_{\mathrm{C}}=52 \mathrm{~nm}$ and $R_{\mathrm{C}}=47 \mathrm{~nm}$ placed in air (black curves) and in paraffin/RTV (blue curves) respectively.

cylinder modes were obtained by solving equation (1) (see section 2) analytically, taking the refractive index of GaAs fixed at the real value corresponding to the laser wavelength ( $n=3.86$ for $\lambda=632.8 \mathrm{~nm}$ ). In figure 6(a), the cylinder radius values ( $Y$-axis) and $k_{z}$ data ( $X$-axis) are multiplied by the laser wavevector $(\omega / c)$ and the related radius, respectively. This allows data for different radiuses to be shown on the same graph. The numerical dispersion data (symbols) show good correspondence with the analytical dispersion curves only around the regions of effective coupling of light into the HE11 and HE12 modes (regions of large variation of modal group velocity), exactly where the resonance excitation peaks are observed in figure 5(a). This is illustrated in figure 6(a) by plotting the $k_{z}$ data symbols with the colour proportional to the FT peak intensity. By following the guiding HE11 mode curve, the dispersion curve for the $4 \mu \mathrm{m}$ long cylinder deviates from the light line by only $\sim 5 \%$ in the $k_{z}$ dimension. For the $2 \mu \mathrm{m}$ long cylinder, the finite-cylinder dispersion curve extends to higher $k_{z}$ values, but the deviation from the light line is no more than $10 \%$ for either HE11 or HE12 resonances. Between the resonances, the numerical dispersion curves return to the light line (plane wave in air), indicating strong deviation from the guiding modes of the infinite cylinder there. Thus, the parallel excitation of a finite cylindrical NW does effectively excite HE1X-like cylinder modes, but only with very small $k_{z}$ vectors. For such modes, the electromagnetic field is still largely distributed outside the NW. Assuming a 10\% deviation from the light line, the effective modal refractive index for the excited HE11 mode is just $\sim 1.10$, which is well below the $n_{\mathrm{c}}=3.86$ value for the case of the modal field being fully confined inside the NW (light line in GaAs). Tapering is expected to further facilitate light coupling into the finite cylinder, which was confirmed by simulation (figure S14), but the deviation from the light line remains very small $(<20 \%)$. 
A similar mode dispersion analysis was also conducted for the cylindrical NW encapsulated in paraffin/RTV. The encapsulating media were simulated as non-absorbing and isotropic with a refractive index of $n_{\mathrm{m}}=1.5$, which is a good approximation for both paraffin $\left(n_{\mathrm{m}}=1.467\right)$ and RTV ( $n_{\mathrm{m}}=1.4$ and 1.5 , depending on composition). Figure $6(\mathrm{~b})$ compares the HE11 mode dispersion for a $4 \mu \mathrm{m}$ long GaAs cylinder in air and in paraffin/RTV media. Because the activated resonance HE11 mode lies very close to the free light dispersion line in relevant media, it shifts with this line as the media refractive index is changed, as indicated by the red arrow (figure 6(b)). This results in approximately $n_{\mathrm{m}}$ time increase in the modal $k_{z}$ wavevector. Additionally, the resonance radius changes from $R_{\mathrm{C}} \omega / c=0.52\left(R_{\mathrm{C}}=52 \mathrm{~nm}\right)$ to $R_{\mathrm{C}}$ $\omega / c=0.47\left(R_{\mathrm{C}}=47 \mathrm{~nm}\right)$. These findings will be reflected in the wavevector conservation analysis below.

\subsection{SPhP dispersion}

To calculate the expected SPhP frequencies in our Raman spectra we adopted a model where both incident and scattered light are propagating as HE11 modes with the $k_{z}$ parameters indicated in figures 6(a) and (b). The dispersion of SPhP modes is calculated in the approximation of an infinite non-adsorbing GaAs cylinder by solving equation (1) with the cylinder dielectric function represented as

$$
\varepsilon(\omega)=\varepsilon_{\infty}+\frac{\varepsilon_{0}-\varepsilon_{\infty}}{1-\left(\frac{\omega}{\omega_{\mathrm{TO}}}\right)^{2}},
$$

where $\varepsilon_{0}$ is the static permittivity, $\varepsilon_{\infty}$ is the permittivity at high (optical) frequencies, and $\omega_{\mathrm{TO}}$ is the TO lattice vibration for zinc-blende GaAs. Taking $\omega_{\mathrm{TO}}=269.3 \mathrm{~cm}^{-1}, \varepsilon_{0}=12.86$, $\varepsilon_{\infty}=10.9$ (equivalent to $\omega_{\mathrm{LO}}=292.5 \mathrm{~cm}^{-1}$ ), the dispersion of the first four SPhP modes $\left(\mathrm{SO}_{0}-\mathrm{SO}_{3}\right)$ has been calculated for the cylinder radius $R_{\mathrm{C}}=52.5 \mathrm{~nm}$ (HE11 resonance) and the results are shown in figure 6(c). We are interested mostly in the lowest symmetrical $\mathrm{SO}_{0}$ mode, which is expected to produce a dominant contribution to the Raman signal $[33,60,61]$.

To calculate the $k_{z} \mathrm{SO}$ value of $\mathrm{SO}_{0}$ mode involved in the backscattering Raman geometry, we have taken the HE11 modal $k_{z} \approx 2 \pi / \lambda_{\mathrm{L}}$ (see figure 6(b)) value twice, ignoring the $k_{z}$ deviation from the light line and the difference in $k_{z}$ between incident and scattered light. For that $k_{z}{ }^{\mathrm{SO}}$, the diagram in figure $6(\mathrm{c})$ gives $\omega_{\mathrm{SO} 0}=287.6 \mathrm{~cm}^{-1}$. This value is in good agreement with the experimentally obtained SO position values of $288 \pm 0.6 \mathrm{~cm}^{-1}$ for the hot-spot NWs of round crosssection.

To find the expected red-shift of the $\mathrm{SO}_{0}$ peak under paraffin/RTV encapsulation, the SO dispersion diagram should be recalculated for a new HE11 resonance condition indicated by the red arrow in figure 6(b). The new condition reflects two factors. First, the encapsulation affects the distribution of the resonant NW radiuses participating in the hotspot assembly. Indeed, the HE11 peak of hot-spot distribution moves from $R_{\mathrm{C}} \approx 52 \mathrm{~nm}$ in air to $R_{\mathrm{C}} \approx 47 \mathrm{~nm}$ in paraffin/RTV (see figure
S12(d)). This factor, together with the change in dielectric media, causes a downshift of all the dispersion curves of SO phonons as shown in figure 6(c). Taken alone, this downshift would translate into an $\mathrm{SO}_{0}$ red-shift of $\approx 5 \mathrm{~cm}^{-1}$ under the encapsulation. However, the encapsulation also increases the $k_{z}$ vector of the resonant HE11 mode by approximately $n_{\mathrm{m}}$ times, shifting the operating point on the $\mathrm{SO}_{0}$ dispersion diagram to higher wavenumbers at $\omega_{\mathrm{SO}} 0=285.3 \mathrm{~cm}^{-1}$ (as shown by the red arrow in figure 6(c)). Thus, the expected $\mathrm{SO}_{0}$ redshift is only about $287.6-285.3=2.3 \mathrm{~cm}^{-1}$, which is in good agreement with the experimental value of 2 and $3 \mathrm{~cm}^{-1}$ (figure 4(f)).

The overall good agreement of the simulated and experimental SO mode parameters confirms the SO mode identification as $\mathrm{SPhP}$.

\section{Conclusions}

A two-orders-of-magnitude enhancement of the overall backscattered Raman signal has been demonstrated in individual GaAs NWs, with the strongest enhancement of the SPhP peak, which became the dominant feature of the spectrum. This brings SPhP Raman spectroscopy to a level suitable for routine practical characterisation of NWs and enables the development of various surface sensors.

The analysis of experimental results supported by extensive numerical simulations established the conditions for the SPhP Raman signal enhancement. It was shown that the enhancement is observed in NWs of a certain diameter range satisfying the condition for resonant enhancement of the incident light intensity inside the NW via excitation of an HE11-like waveguiding mode. This represents an example of dielectric resonance for excitation along the NW axis, which is different from the widely studied Mie resonances. Additionally, NWs should also be tapered to facilitate light coupling with (and decoupling from) the NW and also further concentration of the excitation light inside the NW.

High-amplitude SPhP peaks allowed for immediate evaluation of their polarisation properties, as well as the effects of variation of dielectric environment and the shape of the NW cross-section. In particular, it was revealed for the first time, to our knowledge, that the spectral position and intensity of the SPhP peak both change with the incident light polarisation angle in NWs of elongated cross-section. This finding opens up new prospects for sensor applications.

The high efficiency of the Raman scattering in combination with the waveguiding properties of NWs indicate good prospects for stimulated SPhP Raman scattering in similar sizeresonant nanowires made out of materials with reduced laser absorption.

Since Stokes Raman scattering is also a phonon generation process, the highly efficient SPhP scattering also means there will be efficient generation of high- $k\left(10^{5} \mathrm{~cm}^{-1}\right) \mathrm{SPhPs}$ in an individual NW by the conventional far-field optical method. This implies possible application of the NW as an input port in future low-loss IR photonic microsystems based on high- $k$ SPhPs. 


\section{Data availability statement}

All data that support the findings of this study are included within the article (and any supplementary files).

\section{Acknowledgments}

The authors acknowledge the support of the Leverhulme Trust, EPSRC (Grant Nos. EP/P000916/1 and EP/P000886/1), and the EPSRC National Epitaxy Facility.

\section{Conflict of interest}

The authors declare no competing financial interest.

\section{ORCID iDs}

Sergey I Rybchenko (D) https://orcid.org/0000-0002-9899$313 \mathrm{X}$

Huiyun Liu (D) https://orcid.org/0000-0002-7654-8553

\section{References}

[1] Agranovich V M and Mills D L Modern Problems in Condensed Matter Sciences (ed) 1982 Surface Polaritons: Electromagnetic Waves at Surfaces and Interfaces vol 1 (Amsterdam: North-Holland Publishing Company) p 734

[2] Caldwell J D et al 2015 Low-loss, infrared and terahertz nanophotonics using surface phonon polaritons Nanophotonics 444-68

[3] Berte R, Gubbin C R, Wheeler V D, Giles A J, Giannini V, Maier S A, de Liberato S and Caldwell J D 2018 Sub-nanometer thin oxide film sensing with localized surface phonon polaritons ACS Photonics 5 2807-15

[4] Caldwell J D, Aharonovich I, Cassabois G, Edgar J H, Gil B and Basov D N 2019 Photonics with hexagonal boron nitride Nat. Rev. Mater. 4 552-67

[5] Autore $\mathrm{M}$ et al 2018 Boron nitride nanoresonators for phonon-enhanced molecular vibrational spectroscopy at the strong coupling limit Light Sci. Appl. 717172

[6] Oh S-H and Altug H 2018 Performance metrics and enabling technologies for nanoplasmonic biosensors Nat. Commun. 95263

[7] Caldarola M, Albella P, Cortés E, Rahmani M, Roschuk T, Grinblat G, Oulton R F, Bragas A V and Maier S A 2015 Non-plasmonic nanoantennas for surface enhanced spectroscopies with ultra-low heat conversion Nat. Commun. 67915

[8] Foteinopoulou S, Devarapu G C R, Subramania G S, Krishna S and Wasserman D 2019 Phonon-polaritonics: enabling powerful capabilities for infrared photonics Nanophotonics 8 2129-75

[9] Feng K, Streyer W, Zhong Y, Hoffman A J and Wasserman D 2015 Photonic materials, structures and devices for reststrahlen optics Opt. Express 23 A1418-33

[10] Zou Y, Chakravarty S, Chung C-J, Xu X and Chen R T 2018 Mid-infrared silicon photonic waveguides and devices Photonics Res. 6254

[11] Keilmann F and Hillenbrand R 2004 Near-field microscopy by elastic light scattering from a tip Phil. Trans. R. Soc. A $362787-805$
[12] Hayashi S and Rupin R 1985 Raman scattering from GaP microcrystals: analysis of the surface phonon peak $J$. Phys. C 18 2583-92

[13] Evans D J, Ushioda S and McMullen J D 1973 Raman scattering from surface polaritons in a GaAs film Phys. Rev. Lett. 31369

[14] Davydov V Y, Subashiev A V, Cheng T S, Foxon C T, Goncharuk I N, Smirnov A N and Zolotareva R V 1997 Raman scattering by surface polaritons in cubic $\mathrm{GaN}$ epitaxial layers Solid State Commun. 104 397-400

[15] Nga S S, Yoon T L Hassan Z Abu Hassan H. 2009 Surface and interface phonon polaritons of wurtzite $\mathrm{GaN}$ thin film grown on 6H-SiC substrate Appl. Phys. Lett. 94241912

[16] Dhara S, Sahoo P, Tyagi A K and Raj B 2011 Surface Optical Mode in Semiconductor Nanowires (Rijeka: InTech) pp 3-26

[17] Prasad N and Karthikeyan B 2019 A Raman spectral probe on polar $\mathrm{w}-\mathrm{ZnS}$ nanostructures and surface optical phonon modes in nanowires Nanoscale 11 4948-58

[18] Zhang Y, Wu J, Aagesen M and Liu H 2015 III-V nanowires and nanowire optoelectronic devices J. Phys. D: Appl. Phys. 48463001

[19] Yang P, Yan R and Fardy M 2010 Semiconductor nanowire: what's next? Nano Lett. 10 1529-36

[20] Wong-Leung J, Yang I, Li Z, Karuturi S K, Fu L, Tan H H and Jagadish C 2020 Engineering III-V semiconductor nanowires for device applications $A d v$. Mater. 321904359

[21] Nehra M et al 2020 1D semiconductor nanowires for energy conversion, harvesting and storage applications Nano Energy 76104991

[22] Mata R, Cross A, Hestroffer K and Daudin B 2012 Surface optical phonon modes in $\mathrm{GaN}$ nanowire arrays: dependence on nanowire density and diameter Phys. Rev. B 85035322

[23] García-Núñez C, Braña A F, Pau J L, Ghita D, García B J, Shen G, Wilbert D S, Kim S M and Kung P 2014 Surface optical phonons in GaAs nanowires grown by Ga-assisted chemical beam epitaxy J. Appl. Phys. 115034307

[24] Gupta R, Xiong Q, Mahan G D and Eklund P C 2003 Surface optical phonons in gallium phosphide nanowires Nano Lett. 3 1745-50

[25] Spirkoska D and Abstreiter G Fontcuberta i Morral A 2008 Size and environment dependence of surface phonon modes of gallium arsenide nanowires as measured by Raman spectroscopy Nanotechnology 19435704

[26] Watt M, Sotomayor Torres C M, Arnot H E G and Beaumont S P 1990 Surface phonons in GaAs cylinders Semicond. Sci. Technol. 5 285-90

[27] Walia J, Dhindsa N, Flannery J, Khodabad I, Forrest J, LaPierre R and Saini S S 2014 Enhanced photothermal conversion in vertically oriented gallium arsenide nanowire arrays Nano Lett. 14 5820-6

[28] Wu J, Gupta A K, Gutierrez H R and Eklund P C 2009 Cavity-enhanced stimulated raman scattering from short GaP nanowires Nano Lett. $93252-7$

[29] Speiser E, Hinrichs K, Prete P, Lovergine N and Esser N 2015 Vibrational Raman scattering from surfaces of III-V semiconductors: microscopic and macroscopic surface modes Phys. Status Solidi b 252 11-8

[30] Ho C, Varadhan P, Wang H, Chen C, Fang X and He J 2016 Raman selection rule for surface optical phonons in $\mathrm{ZnS}$ nanobelts Nanoscale 8 5954-8

[31] Im H S, Jung C S, Park K, Jang D M, Lim Y R and Park J 2014 Band gap tuning of twinned GaAsP ternary nanowires J. Phys. Chem. C 118 4546-52

[32] Zardo I, Conesa-Boj S, Peiro F, Morante J R, Arbiol J, Uccelli E, Abstreiter G and Fontcuberta I Morral A 2009 Raman spectroscopy of wurtzite and zinc-blende GaAs 
nanowires: polarization dependence, selection rules, and strain effects Phys. Rev. B 80245324

[33] Englman R and Ruppin R 1968 Optical ionic vibrations in finite ionic crystals J. Phys. C 1 1515-31

[34] Ruppin R and Englman R 1970 Optical phonons of small crystals Rep. Prog. Phys. 33 149-96

[35] Chen G, Wu J, Lu Q, Gutierrez H R, Xiong Q, Pellen M E, Petko J S, Werner D H and Eklund P C 2008 Optical antenna effect in semiconducting nanowires Nano Lett. 8 1341-6

[36] Snyder A W and Love J 1983 Optical Waveguide Theory (Berlin: Springer)

[37] Bohren C F and Huffman D R 1983 Absorption and Scattering of Light by Small Particles (New York: Wiley)

[38] Kuznetsov A I, Miroshnichenko A E, Brongersma M L, Kivshar Y S and Luk'yanchuk B 2016 Optically resonant dielectric nanostructures Science $\mathbf{3 5 4}$ aag2472

[39] Alessandri I and Lombardi J R 2016 Enhanced Raman scattering with dielectrics Chem. Rev. 116 14921-81

[40] Ruppin R 1998 Electromagnetic energy inside an irradiated cylinder J. Opt. Soc. Am. A 15 1891-5

[41] Wang B and Leu P W 2012 Tunable and selective resonant absorption in vertical NWs Opt. Lett. 37 3756-8

[42] Abujetas D R, Paniagua-Domínguez R and Sánchez-Gil J A 2015 Unraveling the Janus role of Mie resonances and leaky/guided modes in semiconductor nanowire absorption for enhanced light harvesting ACS Photonics 2 921-9

[43] Staude I and Schilling J 2017 Metamaterial-inspired silicon nanophotonics Nat. Photon. 11 274-84

[44] Frizyuk K, Hasan M, Krasnok A, Alú A and Petrov M 2018 Enhancement of Raman scattering in dielectric nanostructures with electric and magnetic Mie resonances Phys. Rev. B 97085414

[45] Panda J K, Roy A, Singha A, Gemmi M, Ercolani D, Pellegrini V and Sorba L 2013 Internal field induced enhancement and effect of resonance in Raman scattering of InAs nanowires Solid State Commun. 160 26-31

[46] Cao L, Nabet B and Spanier J E 2006 Enhanced Raman scattering from individual semiconductor nanocones and nanowires Phys. Rev. Lett. 96157402

[47] Lopez F J, Hyun J K, Givan U, Kim I S, Holsteen A L and Lauhon L J 2012 Diameter and polarization-dependent Raman scattering intensities of semiconductor nanowires Nano Lett. 12 2266-71
[48] Agarwal D, Yoo J, Pan A and Agarwal R 2019 Cavity engineering of photon-phonon interactions in $\mathrm{Si}$ nanocavities Nano Lett. 19 7950-6

[49] Sistani M, Bartmann M G, Güsken N A, Oulton R F, Keshmiri H, Luong M A, Robin E, Den Hertog M I and Lugstein A 2020 Stimulated Raman scattering in Ge nanowires J. Phys. Chem. C 124 13872-7

[50] Raha S, Mitra S, Kumar Mondal P, Biswas S, D Holmes J and Singha A 2020 Probing dipole and quadrupole resonance mode in non-plasmonic nanowire using Raman spectroscopy Nanotechnology 31425201

[51] Crark G D and Holonyak N 1967 Optical properties of gallium arsenide-phosphide Phys. Rev. 156913

[52] Aspnes D E and Studna A A 1983 Dielectric functions and optical parameters of $\mathrm{Si}, \mathrm{Ge}, \mathrm{GaP}, \mathrm{GaAs}, \mathrm{GaSb}, \mathrm{InP}$, InAs, and InSb from 1.5-6.0 eV Phys. Rev. B 27 985-1009

[53] Stratton J A 1941 Electromagnetic Theory (London: McGraw-Hill)

[54] Holm J, Jørgensen H I, Krogstrup P, Nygård J, Liu H and Aagesen M 2013 Surface-passivated GaAsP single-nanowire solar cells exceeding $10 \%$ efficiency grown on silicon Nat. Commun. 41498

[55] Zhang Y and Liu H 2020 Self-catalyzed GaAs (P) nanowires and their application for solar cells J. Phys. D: Appl. Phys. 53233001

[56] Pages O, Souhabi J, Postnikov A V and Chafi A 2009 Percolation versus cluster models for multimode vibration spectra of mixed crystals: GaAsP as a case study Phys. Rev. B 80035204

[57] Moore W J and Holm R T 1996 Infrared dielectric constant of GaAs J. Appl. Phys. 806939

[58] Rybchenko S I, Ali S, Zhang Y and Liu H 2021 Polarization properties of Raman scattering by surface phonon polaritons in GaAsP nanowires J. Phys. D: Appl. Phys. (https://doi.org/10.1088/1361-6463/ac2400) Accepted

[59] Smolka F M and Hill H A 1977 Room temperature vulcanizing silicone rubber as an optical element Appl. Opt. 16 292-3

[60] Ruppin R 1975 Thermal fluctuations and Raman-scattering in small spherical crystals J. Phys. C 81969

[61] Ruppin R and Englman R 1969 Rayleigh and Raman Scattering by Surface Modes in Ionic Crystals Light Scattering Spectra of Solids ed G B Wright (New York University, New York, September 3-6, 1968) pp 157-66 